concentrating hormone increases the synaptic interactions between the cardiac sac and gastric mill neurons to such an extent that the entire gastric mill fires with a cardiac sac rhythm. Virtually all of the gastric neurons must be switching to allow the operationally independent gastric mill and cardiac sac central pattern generators to act as one functional unit.

The idea of sharing neurons between networks may confer some as yet unexplainable computational advantage on small systems. It adds further complexity to a specific well-defined system already shown to be extremely versatile in its response to modulators. Moreover, it suggests caution in interpreting the data from single cells of more complex systems; if the chemical environment can switch a neuron from one circuit to another, the hypercomplex cell of one preparation may be the simple cell of another.

Allen Selverston is in the Department of Biology, University of California, San Diego, La Jolla, California 92093, USA.

\title{
Antarctic differences of opinion
}

\section{P. Wright and Monica M. Grady}

THE flux of interplanetary debris delivers meteorites more or less uniformly to all parts of the Earth's surface, yet there is evidence to suggest that the meteorites collected in Antarctica represent a different population from that found elsewhere. Such evidence was presented at a recent meeting* to discuss this distinction, and there was much debate as to whether any differences were real or artefacts. Those with partisan views were unlikely to have changed their opinions about the meaning of the apparent differences between Antarctic and non-Antarctic meteorites in the light of presentations at the meeting. But the idea that the two sample sets may represent some sort of difference in meteorite sources remains a fascinating possibility. The effects of terrestrial weathering must first be constrained, however, if the differences are to be attributed to preterrestrial phenomena.

The first evidence of a distinction was presented by M. E. Lipschutz and colleagues (J. E. Dennison et al. Nature 319, $390-393 ; 1986)$ on the basis of volatile/ mobile trace-element compositions of H5 ordinary chondrites collected from Antarctica and elsewhere. Another apparent difference reported since is that certain meteorite types have thus far only been found in Antarctica. These include polymict brecciated eucrites, lunar meteorites and low $\mathrm{FeO}$ ureilites (the last of which also have distinctive oxygen isotope compositions). Antarctic and nonAntarctic carbonaceous chondrites show differences in chemistry, oxygen isotope composition, extent of aqueous alteration and heating; these effects are undoubtedly due to preterrestrial processes. But do the observed differences document separate meteorite populations or do the data simply extend the currently known ranges in the properties of individual meteorite groups?

Another difference between Antarctic and non-Antarctic meteorites is the higher

* Differences Between Antarctic and Non-Antarctic Meteorites, University of Vienna, 27-28 July 1989 ratio of $\mathrm{H}$ - to L-group chondrites in the Antarctic collection. On closer examination, however, the enhanced ratio is the result of an excess of $\mathrm{H}$-group chondrites from just one Antarctic collection area. Thus, the recent addition of a single shower fall of $\mathrm{H}$-group chondrites to this region would distort the $\mathrm{H} / \mathrm{L}$ ratio of Antarctic meteorites, which would otherwise be similar to that from the rest of the world (G. Huss, University of Chicago). Critics of this hypothesis include $\mathrm{R}$. Harvey (University of Pittsburgh), who warned that the shower would have had to be anomalous to explain the relatively large numbers of small fragments present in the Antarctic collection. The continued recovery of meteorites from Antarctica should help to settle this debate.

If the distinctions are real, what are the reasons? Are the differences the result of preterrestrial processes, so that they can yield interesting clues about the Solar System, as Lipschutz (Purdue University) maintains? Or is a mixture of selection and weathering effects at work? An important distinction between Antarctic and nonAntarctic meteorites is the range of their terrestrial ages: samples collected from observed falls are no more than about 200 years old; by contrast, Antarctic finds may have fallen over the past million years. It is not expected, however, that the parent population has changed over this period. The temporal decay of a meteorite flux, attributable to collisions in the asteroid belt, takes $10^{7}$ years $(\mathrm{G}$. W. Wetherill Nature 319, 357-358; 1986).

Also, the mean size of Antarctic meteorites is much less than that of nonAntarctic samples. Clearly, this is because small fragments are more readily seen when exposed on ice rather than elsewhere. But frequently, numerous small fragments originate from a single fall that broke up in the atmosphere, on impact or subsequently. Thus, although collections include more Antarctic meteorites than non-Antarctic, statistical analyses should rely on their total mass, and not their number, to be reliable.

Complications arise from loss mechanisms that must operate in the Antarctic, the effects of which are not fully understood: small fragments are blown away by the wind, and large fragments may sink into the ice. Furthermore, the collected mass for non-Antarctic meteorites can be much less than that which originally struck the atmosphere. For example, the Revelstoke fireball caused huge atmospheric detonations and the impact was recorded by seismographs, yet the collected meteorite amounted to only 1 gram. Similarly, immediately following a meteor shower over India, only a few large pieces were collected. Over the following three years, however, a coordinated search with the assistance of school children expanded the collection by several hundred pieces.

The idea that the differences are preterrestrial is not supported by the cosmicray exposure ages of $\mathrm{H}$-group chondrites (L. Schultz, Max-Planck-Institut für Chemie), by their major- and minorelement chemistries (E. Jarosewich, Smithsonian Institution), or by their indigenous carbon content and isotope composition (M. M. G.), none of which vary between Antarctic and non-Antarctic samples. If the meteorites are from different sources, then the first finding would imply that two independent source bodies broke up by chance at the same time to supply the putative separate populations.

A possible explanation of the chemical and isotope differences that do exist is terrestrial weathering. Unfortunately, the nature of terrestrial weathering is not well understood: M. A. Velbel (Michigan State University) demonstrated the ineffectiveness of the weathering categories used by NASA's Antarctic meteorite curatorial facility. The modest improvement in weathering classification (to indicate the presence of surficial evaporite deposits) seems a long way short of an adequate description of the weathering histories of Antarctic meteorites. It is difficult to conceive a convenient and rapid screening procedure (over 1,000 meteorite fragments were collected in the US collection programmes in the 1988-89 season alone). Furthermore, the effects of weathering might extend to considerable depth within a meteorite sample (D. W. Mittlefehldt, NASA Johnson Space Center, Houston). Researchers who analyse 'interior' samples should be aware that such materials are often obtained by simply breaking open a meteorite - it is probable that fracturing takes place along pre-existing cracks, which may have been inundated with terrestrial fluids.

1. P. Wright and Monica M. Grady are in the Department of Earth Sciences, The Open University, Walton Hall, Milton Keynes MK7 6AA, UK. 